\title{
miRNA-544a Regulates the Inflammation of Spinal Cord Injury by Inhibiting the Expression of NEUROD4
}

\author{
Lei Yang ${ }^{a} \quad$ Dawei Ge $^{b} \quad$ Xi Chen ${ }^{a} \quad$ Chunzhi Jiang $^{a} \quad$ Shengnai Zheng ${ }^{a}$ \\ aDepartment of Orthopaedics, Nanjing First Hospital, Nanjing Medical University, Nanjing, bepartment \\ of Orthopaedics, the First Affiliated Hospital of Nanjing Medical University, Nanjing, China
}

\section{Key Words}

Spinal cord injury $\cdot \mathrm{miR}-544 a \cdot N E U R O D 4 \cdot$ Inflammation

\begin{abstract}
Background/Aims: To explore the potential role of miR-544a in spinal cord injury and the possible mechanism involved. Methods: We established a mouse model with spinal cord injury to examine the changes in grip force recovery of the forelimb or the posterior limb of the mouse. Microarray was performed to achieve differentiated miRNAs in the mice. The expressions of miR-544a, MCP-1, IL36B and IL17B after spinal cord injury were detected by qRT-PCR. Subsequently, miR-544a was overexpressed to observe changes in inflammation and grip strength after spinal cord injury. Target gene of miR-544a was then predicted using bioinformatics technology. Finally, dual luciferase reporter gene assay was used to verify the binding of miR-544a to its target gene. Results: Using mice models with spinal cord injury, we found that the strength of their four limbs began to recover 7 days after injury. The results of microarray and qRT-PCR confirmed that mir-544a level in mice with spinal cord injury decreased with increase of injury time, while the levels of inflammatory genes MCP-1 (monocyte chemoattractant protein-1), IL1 (interleukin-1) and TNF- $\alpha$ (tumor necrosis factor alpha) IL36B (interleukin-36 beta) and IL17B (interleukin-17 beta) were significantly increased. However, overexpression of miR-544a in the mice significantly reduced the level of inflammation and restored their grip strength in their four limbs. Finally, we found that miR-544a can bind to the NEUROD4 (Neurogenic differentiation 4) 3'UTR (Untranslated Region) region through bioinformatics website prediction, which was further confirmed by dual luciferase reporter assay. NEUROD4 level was significantly reduced following the overexpression of miR-544a. Conclusion: The expression of miR-544a was significantly decreased after spinal cord injury. High expression of miR-544a could alleviate the inflammation caused by spinal cord injury and promote the recovery of spinal cord via the inhibition of NEUROD4.
\end{abstract}

L. Yang, D. Ge and X. Chen contributed equally to this work. 


\section{Cellular Physiology Cell Physiol Biochem 2018;51:1921-1931

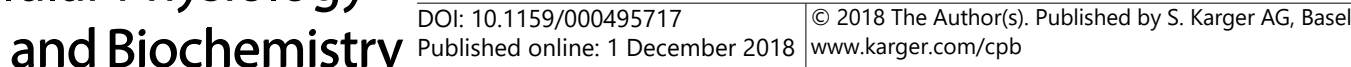 \\ Yang et al.: miR-544a is Involved in the Development of Spinal Cord Injury}

\section{Introduction}

Spinal cord injury (SCI) is caused by the direct or indirect strike from external forces on the spinal cord. Prognosis of patients with SCI is very poor, leading to limb movement disorders, loss of cognitive function or even paralysis, which brings great pain and heavy burden to patients and their families. The incidence of SCI has been increasing year by year. Statistics show that there are 11,000 new cases of SCI each year in the United States [1] and more than 60, 000 in China. In the past, medical scientists thought that the ability of spinal cord to repair itself could not be possible. A study in 1980s however, revealed that nerve cell regeneration could promote SCI recovery [2]. Inflammatory reaction after SCI is the most important pathological process of secondary injury, and inflammatory cytokines are the basic effector molecules, which participate in and regulate the inflammatory response after SCI. Inflammatory cytokines after SCI regulate nerve regeneration and prognosis [3]. Expression of inflammatory cytokines after SCI is not static, they are with the development of SCI showed different changes. Thus to understand local inflammatory factors in the injury area after SCI such as proinflammatory cytokines, chemokines and changes in antiinflammatory factors are of great significance [4].

Many studies have shown that the expression level and post-transcriptional regulation of many genes play an important role during the development of SCI [5, 6]. As miRNAs play important role in post-transcriptional regulation, their regulatory role has been increasingly appreciated by researchers [7, 8]. MiRNAs are 20-24 nucleotides in length and were first discovered in Caenorhabditis elegans in 1993[9]. In humans, miRNAs can bind to the 3'UTR of the target mRNA to promote degradation or inhibit the translation process so as to regulate the gene expression at the post-transcriptional level. The cellular regulation by miRNA, therefore, is an ubiquitous intracellular way of gene regulation. It was found that multiple miRNAs can regulate an identical gene and one miRNA is also able to coordinate the post-transcriptional regulation of multiple genes. It is predicted that miRNAs regulate the expression of nearly one-third of human genes [10].

Many studies have demonstrated the expression patterns and functional characteristics of miRNAs in the process of SCI [11]. It was demonstrated that the severity of SCI is closely related to the level of differentially related genes, which is higher in mild and moderate damage compared to severe injury. Although miRNAs are continually studied, their role in SCI and mechanism involved is yet to be demonstrated [12]..

\section{Materials and Methods}

\section{SCI modeling and mice grouping}

Thirty ten-week-old female C57bl / 6J mice weighing 20-25g were selected for the study. All of them were bred under a normal light cycle and fed with normal diet. They were randomly divided into 2 groups using random number generator. Mice in injury group (SCI group) were made with contusion on the fifth cervical spinal cord. C4-6 spinous processes were revealed by longitudinal incision in the neck and C5 lamina was excised with an ophthalmic scissors to show the dural sac. Hammer position of spinal impactor was adjusted on the C5 spinal cord, and then bleeding was stopped and the wound sutured. For the mice in sham group, simple laminectomy was performed following the same procedure as the SCI group except the last impact on C5 spinal cord. Subsequently, the situation of spinal cord injury was evaluated before or at 2 days, 8 days, 12 days, 21 days, 26 days, 33 days, 41 days, and 52 days after injury. Injured mice were sacrificed to collect their spinal cord specimens at 1 day, 3 days, 5 days, 7 days and 20 days after SCI. This study was approved by the Animal Care Committee of Nanjing Medical University. All protocols of animal experiments were performed according to the Guide for the Care and Use of Laboratory Animals proposed by the Chinese National Institutes of Health. 


\section{Cellular Physiology Cell Physiol Biochem 2018;51:1921-1931 \begin{tabular}{l|l|l} 
and Biochemistry Published onlıne: 1 December 2018 & $\begin{array}{l}\text { (c) } 2018 \text { The Author(s). Published by S. Karger AG, Basel } \\
\text { www.karger.com/cpb }\end{array}$ \\
\hline
\end{tabular}}

\section{Measurement of forelimb grip strength}

Mice tail was lifted up and got close to grip gauge crossbar in order to induce their ability to grab bar. We then gently pulled mice backwards in the horizontal direction to pull them off the crossbar. Instrument showed grip force of the two forepaws of mice. One of the front paws of mice was wrapped with tape and then the left or right forelimb grip strength was measured using the methods described above. The above grip measurement was repeated four times and the average calculated. When a mouse was unable to grip the crossbar due to severe forelimb dyskinesia in measurement, the grip was recorded as zero.

\section{Spinal cord acquisition, RNA extraction and ChIP detection}

After the mice were sacrificed using $\mathrm{CO}_{2}$ asphyxiation treatment, the dorsal skin was cut and the subcutaneous tissues and muscles were removed. The lamina on section including sample tissue was removed, and $4 \mathrm{~mm}$ spinal cord tissue was excised with the injury area taken as the center. These spinal cord tissue specimens were then put in $1.5 \mathrm{ml}$ centrifuge tubes and stored at $-80^{\circ} \mathrm{C}$.

Spinal cord tissue samples were placed in a mortar and $1 \mathrm{ml}$ of Trizol reagent was added to homogenize the tissue by sonication. The homogenate was transferred to a $1.5 \mathrm{ml} \mathrm{EP}$ tube and allowed to stand at room temperature for 5 minutes. $200 \mu \mathrm{l}$ of chloroform reagent was added to each tube and mixed well and let to stand for 15 minutes at room temperature, then centrifuged for 15 minutes. The supernatant was transferred to a new $1.5 \mathrm{ml} \mathrm{EP}$ tube, and the same volume of pre-cooled isopropanol as the supernatant was added. After centrifugation for 10 minutes, the supernatant was discarded and $1 \mathrm{ml}$ of pre-cooled $75 \%$ ethanol was added to wash the pellet. The residue was aspirated and the pellet was allowed to dry at room temperature. Subsequently, $20 \mu \mathrm{l}$ of enzyme-free water was added to completely dissolve RNA. The extracted RNA concentration was assayed by UV analysis. Total RNA was sent to Beijing Boao for miRNA ChIP detection.

\section{QRT-PCR (Quantitative real-time reverse transcription-polymerase chain reaction)}

cDNA was obtained from extracted RNA using reverse transcription and used for PCR detection with SYBR Green method. The amplification conditions are as follows: pre-denaturation at $94^{\circ} \mathrm{C}$ for $5 \mathrm{~min}$ followed by 40 cycles of $94^{\circ} \mathrm{C}$ for $30 \mathrm{~s}, 55^{\circ} \mathrm{C}$ for $30 \mathrm{~s}$ and $72^{\circ} \mathrm{C}$ for $1 \mathrm{~min} 30 \mathrm{~s}$. The primer sequences used were as follows: IL36B (F: 5'-AGAGTATTCAAATGTGGGAACCG-3 ; R: 5'-GACCCATACCATCTGTTGTGAG-3'), IL17B (F: 5'-CTCCTGCTTCTAGGCTGGTTG-3 ; R: 5'CCACCTGGCACTTCGAGTTAG-3' NEUROD4 (F: 5'-AGCTGGTCAACACACAATCCT-3 ; R: 5'-TTCCATAAGAGCCCGGTCTTC-3'), GAPDH (F: 5'-AATGGATTTGGACGCATTGGT-3 ; R: 5'-TTTGCACTGGTACGTGTTGAT-3'). MiR-544a real-time PCR assay kit was used from Guangzhou Ruibo Biotechnology Co., Ltd. for PCR amplification in order to detect the expression of miR-544a.

Construction of luciferase reporter gene vector and luciferase activity assay

The NEUROD4 wild-type sequence (NEUROD4 WT 3'UTR) and the mutant sequence (NEUROD4 MUT 3'UTR) were constructed based on the 3'UTR sequence of NEUROD4. Cells were then seeded in 24-well plates and co-transfected with miR-544a mimics and NEUROD4 WT, miR-544a mimics and NEUROD4 MUT, Negative Control (NC) and NEUROD4 WT, NC and NEUROD4 MUT 3 'UTRs. 48 hours after transfection, fluorescence intensity of the cells was detected using dual luciferase reporter assay system.

\section{Western blot assay}

The medium in the culture dish was aspirated and the cells were washed twice with PBS and lysed on ice. The cells were transferred to an EP tube and then sonicated. After centrifugation for $30 \mathrm{~min}$, the supernatant was taken and added in Bromophenol blue $(9.5 \mathrm{ml}$ per $0.5 \mathrm{ml}$ of protein supernatant) to boil for $10 \mathrm{~min}$. The protein sample was then aliquoted and stored at $-20{ }^{\circ} \mathrm{C}$. The protein sample was taken out and thawed when used. Each protein sample was loaded in SDS-PAGE gel at 105 volts for $2 \mathrm{~h}$. After the gel was transferred into PVDF membrane, the membrane was blocked, incubated with primary antibodies overnight, and incubated with secondary antibodies. Primary rabbit monoclonal MCP-1 antibody (dilution: 1/500; CatNOs: ab25124); rabbit polyclonal IL1 antibody (dilution: 1/500; CatNOs: ab9614); rabbit polyclonal TNF- $\alpha$ antibody (dilution: 1/500; CatNOs: ab6671); rabbit polyclonal IL17B antibody (dilution: 1/500; CatNOs: ab229320); rabbit polyclonal IL36B antibody (dilution: 1/500; CatNOs: ab116280); rabbit polyclonal NEUROD4 antibody (dilution: 1/500; CatNOs: ab90484); rabbit polyclonal GAPDH antibody 


\section{Cellular Physiology Cell Physiol Biochem 2018;51:1921-1931 \begin{tabular}{ll|l} 
and BiOChemistry & $\begin{array}{l}\text { DOI: 10.1159/000495717 } \\
\text { Published online: 1 December } 2018\end{array}$ & $\begin{array}{l}\text { ( } 2018 \text { The Author(s). Published by S. Karger AG, Basel } \\
\text { www.karger.com/cpb }\end{array}$ \\
\hline
\end{tabular}}

(dilution: 1:500, CatNOs: ab37168) and secondary goat anti-rabbit (HRP) IgG antibody (dilution: 1/2000; CatNOs: ab6721) were all purchased from Abcam (Cambridge, MA, USA). Finally, chemiluminescent film was applied for assessment of protein expression with Image J software.

\section{Statistical analysis}

All experiments were repeated three times. SPSS 22.0 statistical software was used for data analysis and GraphPad for image editing. Measurement data were presented as mean \pm SEM of three independent experiments and assessed by $t$ test. $P$ value $<0.05$ was considered statistically significant.

\section{Results}

The influence of spinal cord injury on the grip strength of mice

To confirm whether the SCI modeling was successful, grip forces of the left and right forelimbs of the mice were detected. During the first week, motor function of the injured limbs was severely impaired and the mice could not use the forepaw for any movement. On the $8 \mathrm{~d}-12 \mathrm{~d}$, the mice tried to grasp measuring rod at the time of measurement, but could not complete the successful grasping. Alternatively, even if they completed the grasping, they were still unable to resist the pulling to complete the measurement. After $21 \mathrm{~d}-52 \mathrm{~d}$, grip strength of the left and/or right forelimbs gradually improved over time so as to complete the measurement, but the reading was very low, indicating the existence of serious dyskinesia. During the early stage, mice in sham-operated group were not able to complete the stretch due to the surgical wound pain, resulting in the temporary decrease in measured value of grip force. One week after the operation, the wound gradually healed and the grip strength recovered to the pre-operative level. The grip strength of the mice in the injury group was lower than that in the sham-operated group at various time points after operation; however, the changes of grip force in the two forelimbs were basically the same (Fig. 1A, 1B and 1C).

Spinal cord injury affects the expression of miR-544a and inflammation-related factors

To explore whether miR-544a significantly altered after SCI, RNA of the spinal cord tissues of mice in the injury group at 3 days, 1 week, 2 weeks and 1 month after operation was sequenced. We found that the expression of miR-544a gradually declined from the first day after operation but was restored at 1 month, which is consistent with the result of grip force test (Fig. 2A). We then verified the expression of miR-544a in SCI mouse model by realtime PCR. The expression of miR-544a was consistent with that in sham-operated group
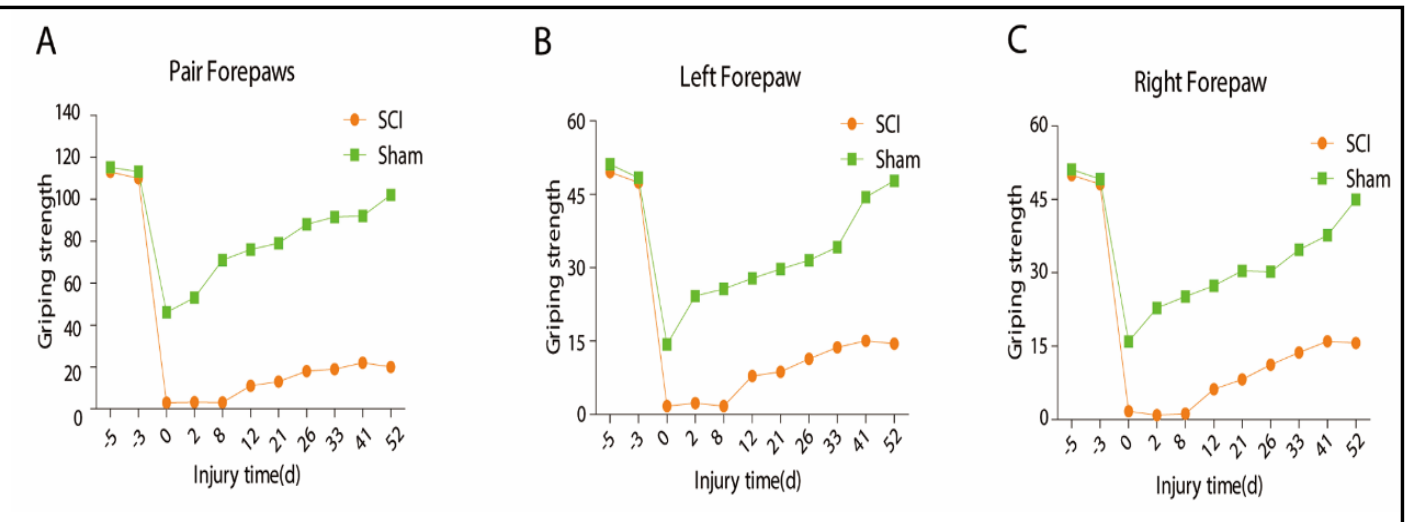

Fig. 1. Measurement results of grip strength in mice with cervical spinal contusion are shown. Grip strength of mice in the pair forepaws (A), left forepaw (B) and right forepaw (C) in the sham group decreased temporarily and gradually recovered to the preoperative level, while mice in the SCI group showed serious dyskinesia. 

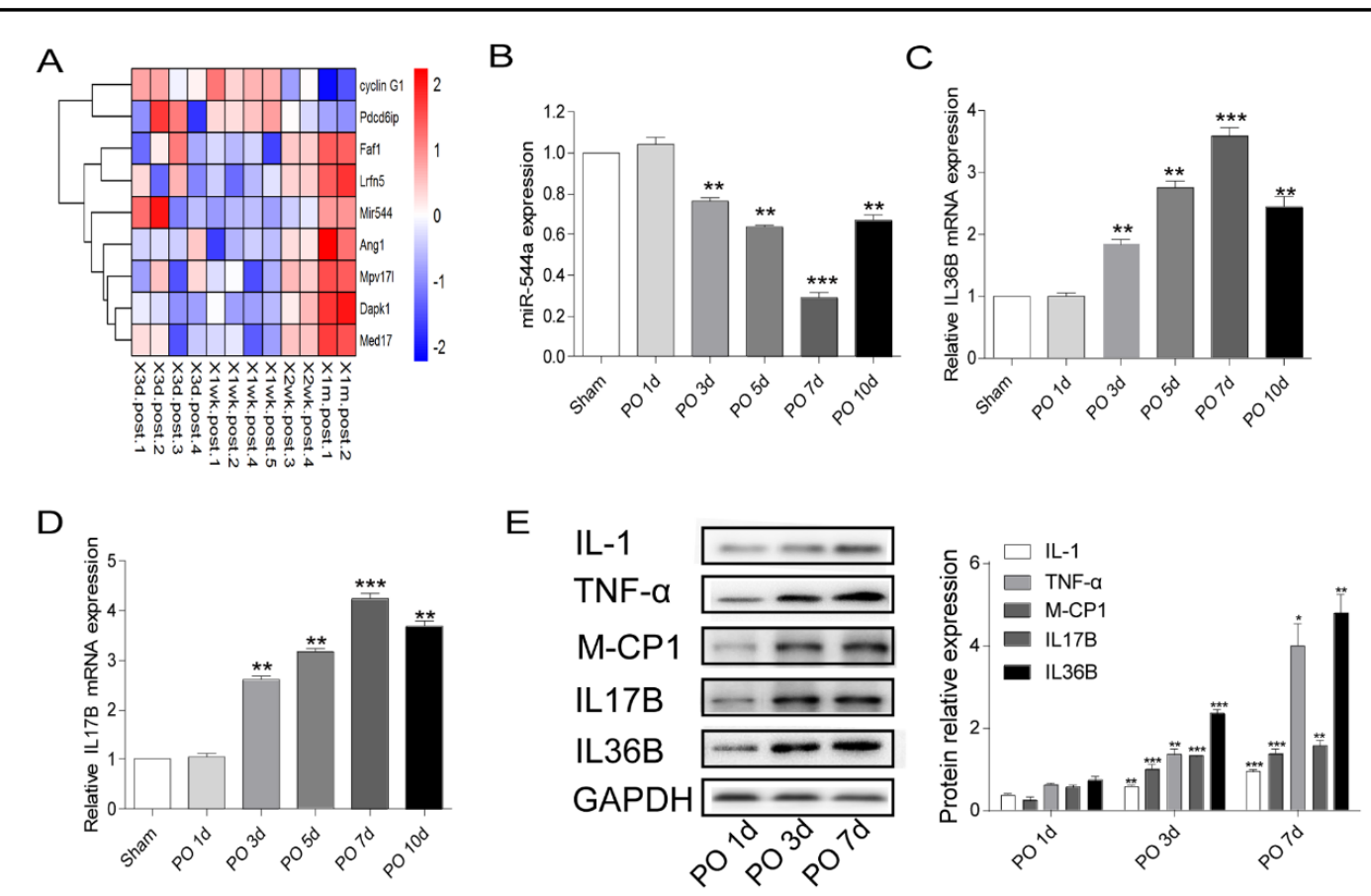

Fig. 2. MiR-544a and inflammation-related factors significantly changed. (A) MiRNAs that expressed differently before and after SCI are presented; (B) MiR-544a level was significantly reduced with the increased injury time and reached the lowest on the 7th day after SCI; The mRNA levels of IL36B (C) and IL17B (D) increased as the injury time increased; (E) Protein expressions of MCP-1, IL1, TNF- $\alpha$, IL36B and IL17B increased with increasing injury time.

one day after operation; however, its expression gradually decreased from the third day and recovered again on the tenth day after surgery (Fig. 2B).

Inflammation after SCI has been demonstrated by many studies as a critical factor in SCI and its recovery, hence we examined the expression of IL36B and IL17B and found that their expression increased with the prolongation of injury time (Fig. 2C and 2D). Further, Western blot results showed that the levels of MCP-1, IL1, TNF- $\alpha$, IL36B and IL17B were enhanced at 1 day, 3 days and 7 days after spinal cord injury with the lesion time extended (Fig. 2E).

The impact of miR-544a overexpression on inflammation.

Next, we investigated the association of miR-544a with levels of inflammatory factors. The expression of miR-544a in the injury area of spinal cord on the first day after surgery of the sham-operated group was taken as a blank control. In the injured group, the expression of miR-544a slightly increased on the first day after injury, began to decrease on the 3rd day and finally reached the lowest value on the $7^{\text {th }}$ day. In the negative control group, the expression trend of miR-544a at each time point after operation showed no significant difference compared to the mice in the injured group, indicating that there was no significant difference between the two groups. However, the miR-544a level in mice of the treated groups injected with exogenous miR-544a was significantly higher than that of the injury group or the negative control group at corresponding time points after operation, with the highest value on the 3rd day (Fig. 3A).

In mice with spinal cord injury, the expressions of IL36B and IL17B mRNAs in each group changed significantly with time. Taking the sham-operated group as the reference, the expressions of IL36B and IL17B mRNAs in the mice of injury group were significantly upregulated at each postoperative time point and reached the highest value on the 7 th day after the operation. However, the expression levels of IL36B and IL17B mRNAs in miR-544a 


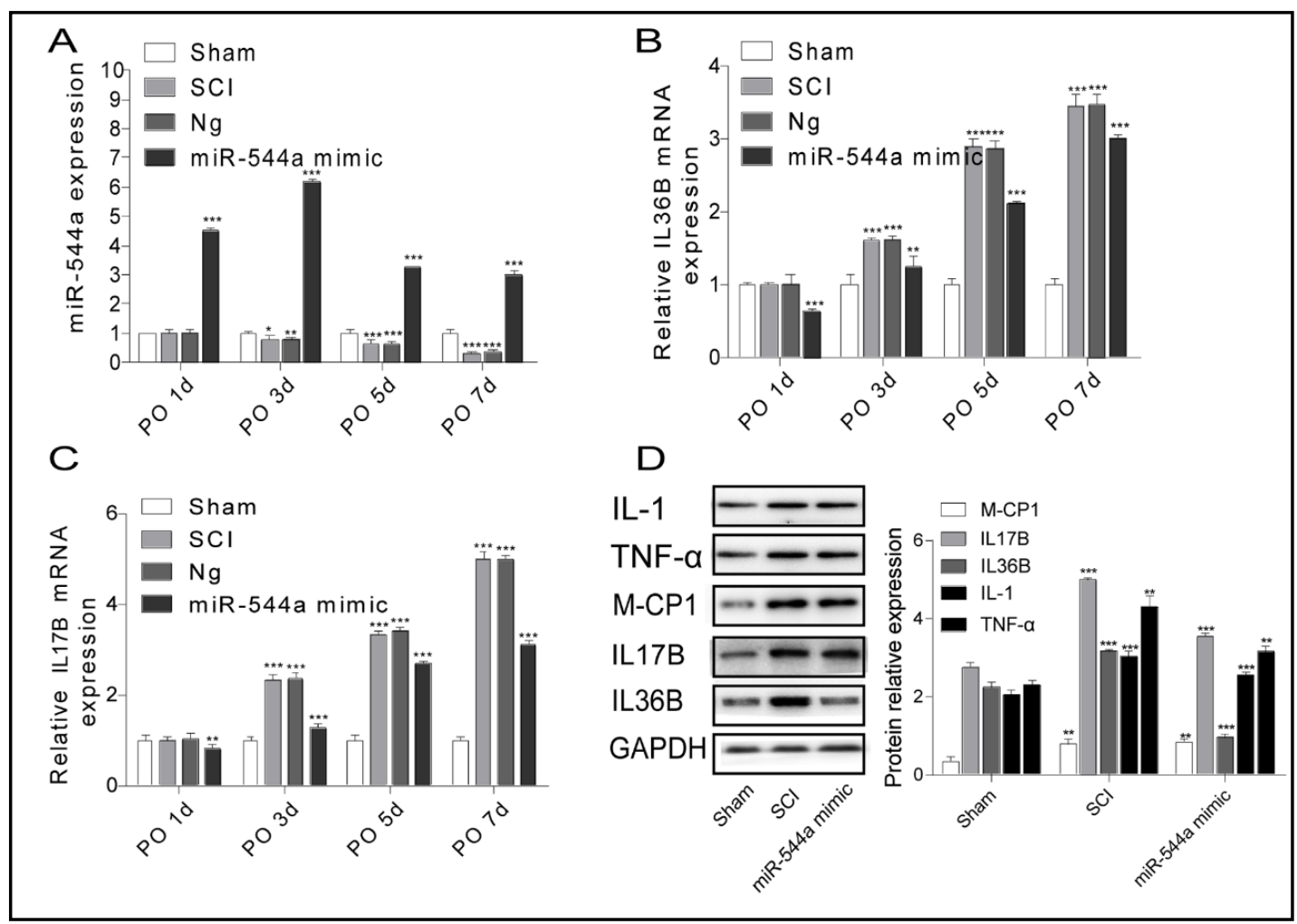

Fig. 3. Effect of overexpression of miR-544a on IL36B and IL17B levels is shown. (A) The expression of miR544a in SCI area of mice in each group is shown.; Influence of overexpressed miR-544a in the injury area of mice on IL36B (B) and IL17B (C) mRNA expression level is presented; (D) Effect of miR-544a overexpression on MCP-1, IL1, TNF- $\alpha$, IL36B and IL17B protein expressions in mice with SCI was shown.

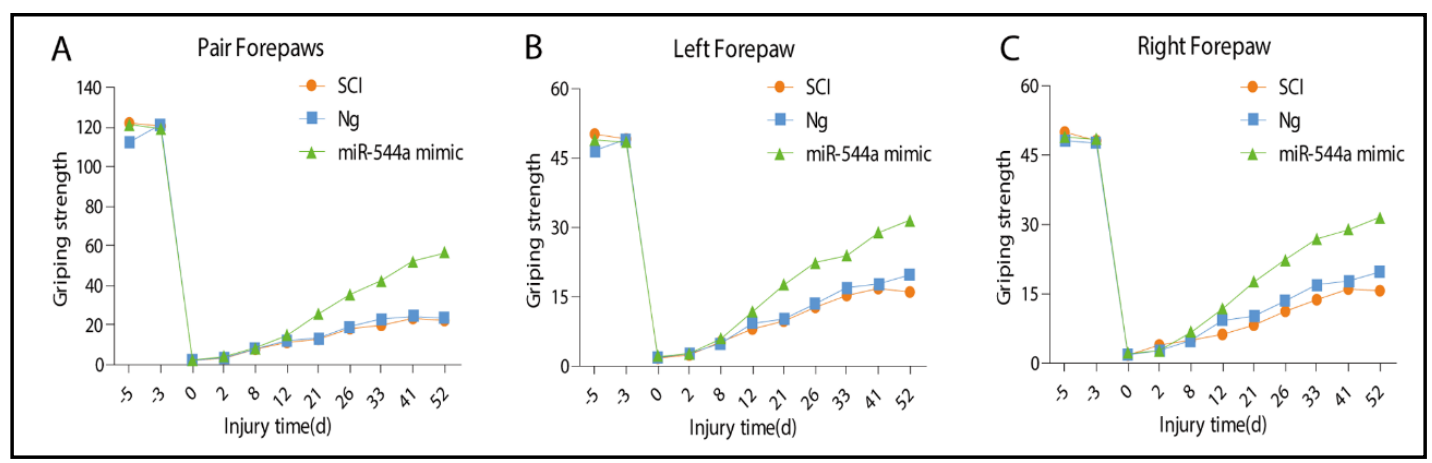

Fig. 4. Effect of miR-544a on reparation and regeneration of spinal cord in mice is presented. (A) Changes in grip force of two forelimbs of mice at different time points after SCI in each group is shown. (B) Changes in grip force of right forearm of mice at different time points after SCI in each group is shown. (C) Changes in left forearm grip force of mice at different time points after SCI in each group is shown.

injection group at each time point after injury were significantly lower than those in injury group at the same time point, indicating that miR-544a overexpression after SCI could induce downregulation of IL36B and IL17B mRNAs in the lesion area (Fig. 3B and 3C). It is an indisputable fact that the expression of various inflammatory factors such as TNF- $\alpha$ is upregulated after SCI, and the inflammatory factors such as IL1 and TNF- $\alpha$ can obviously promote the expression of MCP-1[13]. The western blot results showed that overexpression of miR-544a using mimics significantly decreased the expressions of MCP-1, IL1, TNF- $\alpha$, IL17B and IL36B (Fig. 3D). 


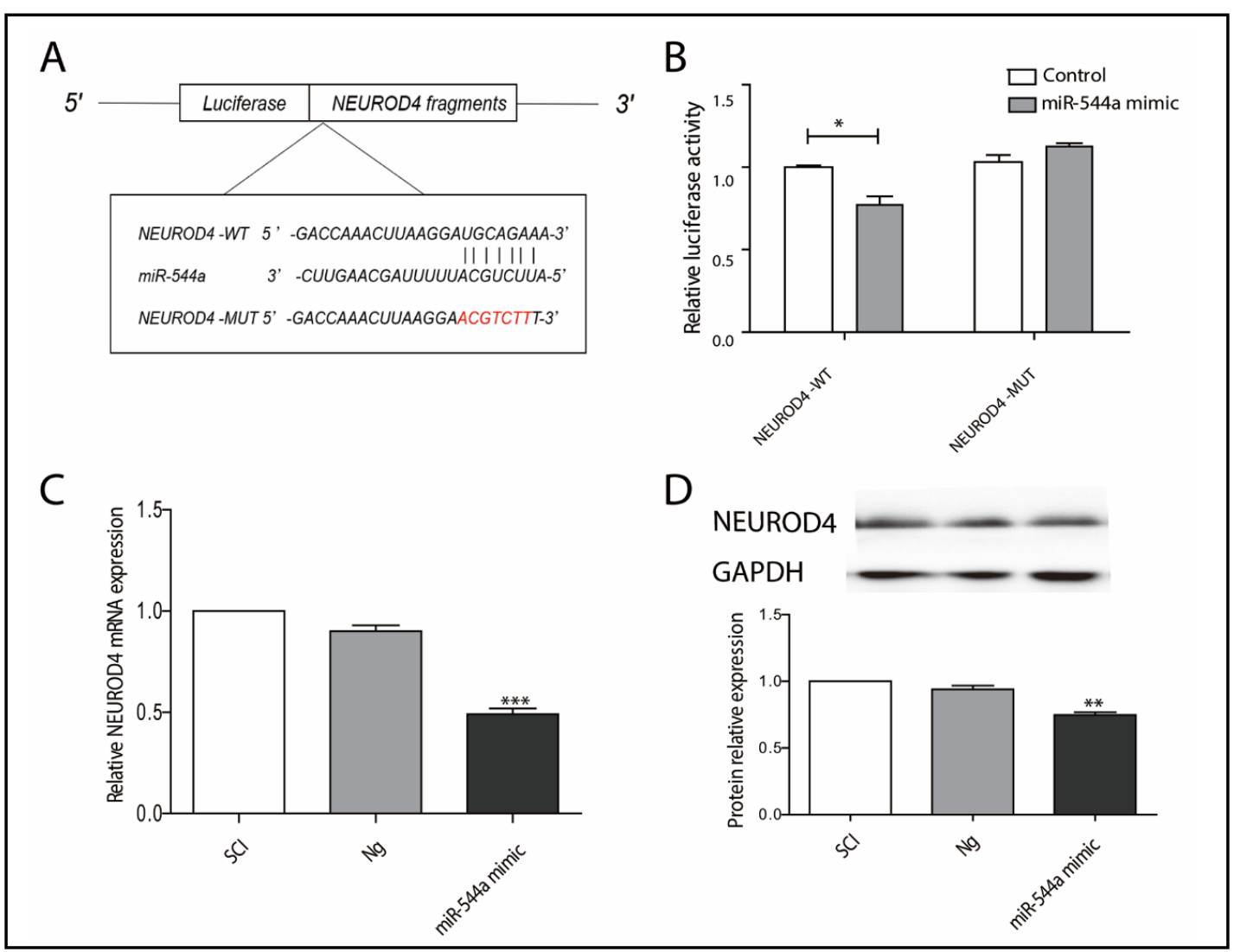

Fig. 5. NEUROD4 was predicted as a target gene for miR-544a. (A) NEUROD4-WT, and NEUROD4-MUT sequences wereas constructed. (B) After the overexpression of miR-544a, luciferase activity decreased in NEUROD4-WT group, while no significant difference was found in NEUROD4-MUT group. (C) Overexpression of miR-544a brought down NEUROD4 mRNA levels. (D) After miR-544a mimic was transfected in, NEUROD4 protein levels decreased.

The effect of miR-544a on the reparation and regeneration of spinal cord injury in mice

Further, to study the effects of miR-544a on the reparation and regeneration of spinal cord injury in mice, forelimb grip strength was measured. Mice with spinal cord injury had serious motor dysfunction of the extremities in the first week, and could not use the forepaw. Mice treated with miR-544a also developed severe dyskinesia after injury. However, forepaw function of some mice with miR-544a overexpression began to recover from the 12th day after injury, and the grip strength was gradually improved with time. In the $21 \mathrm{~d}-52 \mathrm{~d}$, the left and right forepaws grip strength and the two-jaw grip strength of the mice in the treatment group were significantly improved compared with the simple injury group (Fig. 4A, 4B and $4 \mathrm{C})$.

\section{MiR-544a exerts functions by targeting NEUROD4}

To study the further molecular mechanism by which miR-544a regulates the regeneration procedure of spinal cord injury, bioinformatics analysis and dual luciferase reporter gene assay were performed. MiRNAs can set up base pairs with the mRNA of their own target gene to form a gene silencing complex, which degrades the target gene mRNA resulting in pathogenesis. Based on bioinformatics prediction and functional analysis, NEUROD4, a kind of neuronal differentiation factors, was found to be the target gene of miR544a. In order to further verify whether miR-544a can bind to NEUROD4, we constructed NEUROD4 wildtype sequence NEUROD4-WT 3'UTR and mutant sequence NEUROD4-MUT 3'UTR and performed luciferase reporter assay (Fig. 5A). After cells were transfected with 


\section{Cellular Physiology Cell Physiol Biochem 2018;51:1921-1931

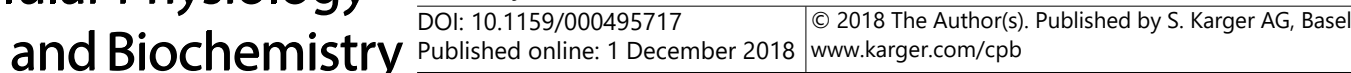

Yang et al.: miR-544a is Involved in the Development of Spinal Cord Injury

miR-544a mimic, luciferase activity in NEUROD4-WT 3'UTR group decreased compared with the negative control group, whereas in the NEUROD4-MUT 3'UTR group it showed no significant difference (Fig. 5B). This implies that miR-544a can interact with NEUROD4. To further verify the binding of miR-544a to NEUROD4, we injected miR-544a mimic into spinal cord tissue and found that the NEUROD4 gene expression was significantly reduced by miR544a overexpression (Fig. 5C). Subsequent testing of protein levels revealed a decrease in NEUROD4 protein expression after miR-544a overexpression (Fig. 5D), demonstrating that miR-544a exerted its effect by inhibiting NEUROD4.

\section{Discussion}

SCI is a very common type of spinal surgery injury [14]. Because impaired sensory motor function is difficult to recover, SCI often leads to significant physical along with psychological trauma and brings financial burdens to patients and their families. The recovery of upper limb function, in particular, is especially vital for patients with cervical spinal cord injury [15].

In recent years, miRNA-related research has revealed its important role during the process of spinal cord development or diseases of spinal cord. Studies confirmed that miRNAs can regulate a variety of transcription factors that play important role during the process of spinal cord development and establishment of functions, including the differentiation of spinal cord neurons and the maintenance of cell type [16-18].

Additionally, the important regulatory role of miRNA in the pathological process of spinal cord injury has also been gradually confirmed. Studies suggest that miRNAs are essential in the regulation of inflammatory signaling pathways and pathological immune responses. Changes in the levels of lots of miRNAs occur after spinal cord injury, which are believed to be involved in immune responses such as invasion of immune cells and regulation of inflammatory signaling pathways [19-21]. In this study, miR-544a was screened by microarray for differential expression levels after spinal cord injury. Meanwhile, levels of inflammation-related genes were also found altered.

MiR-544a is a newly discovered miRNA that is located at $14 \mathrm{q} 32.31$ with few related studies. Previous studies have shown that miR-544a promotes the invasion of lung cancer cells and breast cancer cells by downregulating E-CAD and upregulating vimentin expression $[22,23]$. In addition, miR-544a maintains the self-renewal ability of lung cancer stem cells by downregulating GSK-3 $\beta$ expression [24]. Recent studies have indicated that miR-544a induces EMT phenotype in gastric cancer cells by activating the WNT signaling pathway [25]. Meanwhile, as costimulatory molecules, miR-544a and a cluster of miRNAs located at $14 q 32$, such as miR-127-5p and miR-655-3p, affect multiple metastasis-associated pathways by inhibiting a series of identical target genes [26]. However, the mechanism of miR-544a in spinal cord injury is not clear. Our current study showed that overexpression of miR-544 can alleviate the damage in the grasping ability of mice, indicating that miR-544a plays an important role in the recovery procedure of spinal cord injury.

MCP-1 is an important chemokine that binds to CCR2 (CC chemokine receptor 2), an expressed receptor on the surface of inflammatory cells. MCP-1 is mainly chemotactic to enter infection, injury or inflammation. They are closely related to the changes of axonal demyelination after SCI. Pineau [27] et al. found that astrocytes can produce MCP-1 on monocytes and neutrophils after SCI depend on IL1 receptor / MyD88 path. Perrin [28] et al. administered MCP-1 antibody significantly inhibited the damage of macrophage activity in the injured area, inhibiting the destruction of myelinated debris in the lesion area of SCI. This experiment found that the expression of MCP-1 increased in SCI, but decreased after addition of miR-544a mimics.

NEUROD4, as a neuronal differentiation factor, plays an important role in neuronal cell differentiation. NEUROD is highly expressed during the development of the peripheral and central nervous system; however, its expression levels decrease with the maturation 


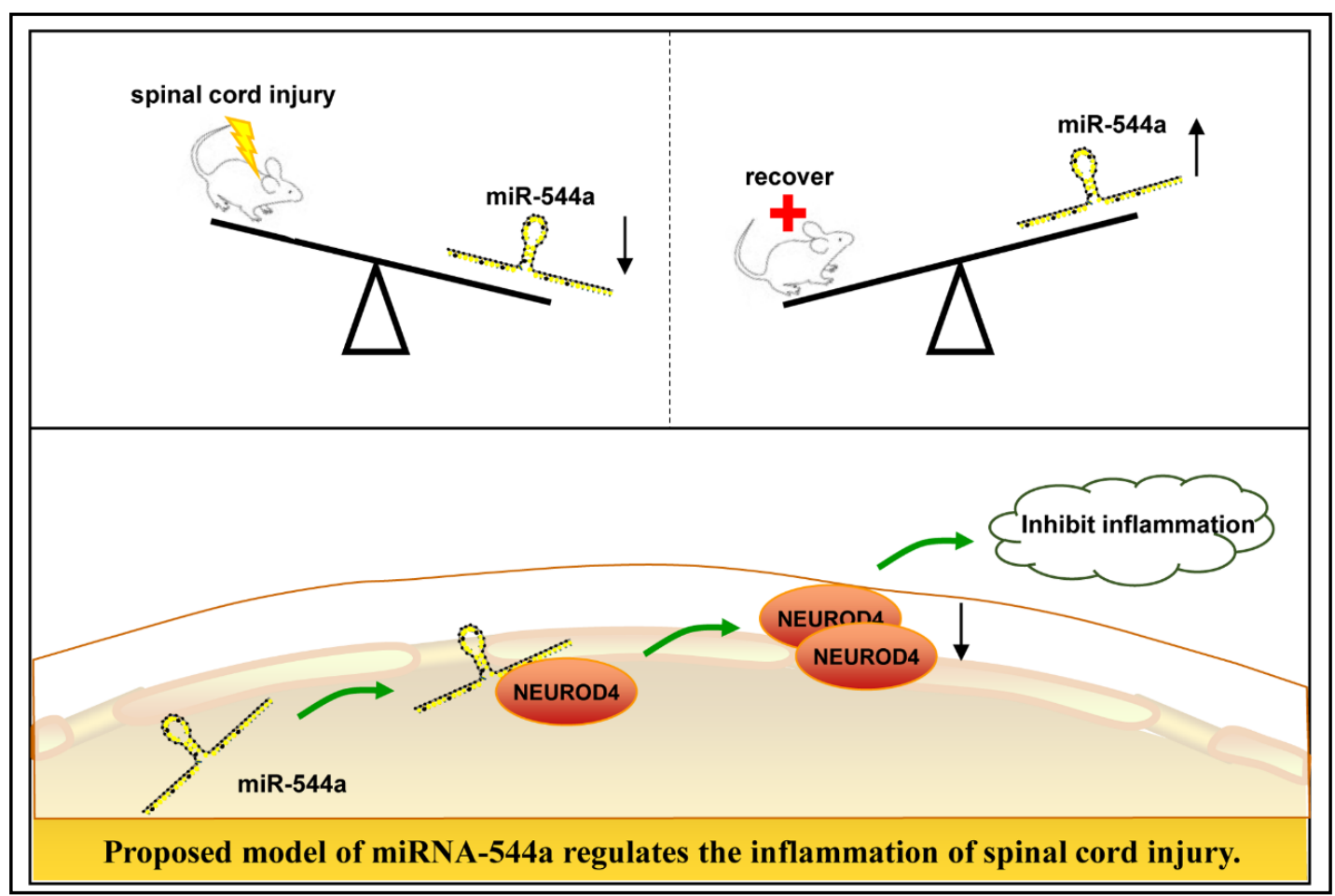

Fig. 6. A proposed model for miR-544a-mediated recovery after SCI. Spinal cord injury leaded to significantly reduction of miR-544a. However, treatment with exogenous miR-544a mimics markedly upregulated miR544a expression, consequently inhibiting the expression of NEUROD4, thus reducing inflammation after SCI.

of neurons. The sustained expression of NEUROD in adult and mouse cerebellum and hippocampus cells may be related to the regeneration of neuronal cells. The NEUROD gene is expressed in 12 kinds of medulloblastomas, neuroblastomas and retinoblastomas, and is significantly upregulated by various stimuli or induction to neuroblasts. In this study, NEUROD4 was identified as the target gene of miR-544a, and its expression was significantly decreased after miR-544a treatment in SCI group [29].

The innovativeness of this study is that miR-544a and its target gene NEUROD4 are both reported for the first time in spinal cord injury. We proposed new clues to the pathogenesis of spinal cord injury and expanded the pathogenesis of spinal cord injury. These findings may provide a new direction for the diagnosis and treatment of spinal cord injury (Fig. 6). However, there are some inadequacies of this study, whether miRNAs could play a role in SCI pathogenesis through the selected target gene requires a more sophisticated proof of reversal. In addition, it is unclear how the abnormal expression of miRNA is regulated. If we can further explore the mechanism of miRNA regulation, it will provide a more in-depth understanding of the pathogenesis of SCI. Thirdly, data regarding the effects of miR-544a knockdown on inflammation of SCI was not provided in this study. This is really a limitation existed in the present study. Actually, we performed knockdown of miR-544a for multiple times using miR-544a inhibitors via caudal vein injection. However, the silencing effects were very poor. Then we also attempted to use Lentivirus to knockdown the expression of miR-544a in vivo again, however, the effects were still not satisfied. In our future study, we have to try our best to find more effective solution to establish the miR-544a knockdown mice models, thus strengthening the significance of our present research. 


\section{Cellular Physiology Cell Physiol Biochem 2018;51:1921-1931 \begin{tabular}{ll|l} 
and BiOChemistry & $\begin{array}{l}\text { DOI: 10.1159/000495717 } \\
\text { Published online: 1 December } 2018\end{array}$ & $\begin{array}{l}\text { ( } 2018 \text { The Author(s). Published by S. Karger AG, Basel } \\
\text { www.karger.com/cpb }\end{array}$ \\
\hline
\end{tabular}}

\section{Conclusion}

In summary, we found that miR-544a was downregulated in mice with spinal cord injury, while inflammation-related genes were highly expressed. Increased miR-544a levels can inhibit inflammatory response in SCI animal model, and to a certain extent promote the recovery of the grip strength of SCI mice. In addition, we verified that miR-544a could act on NEUROD4 to initiate its effects. It is therefore suggestive that miR-544a plays a vital regulatory role in the recovery of spinal cord injury and may be an important target for promoting the repair process.

\section{Acknowledgements}

This study was supported by the National Natural Science Fund from the National Natural Science Foundation of China (Grant No. 81802149).

\section{Disclosure Statement}

The authors declare no conflicts of interest.

\section{References}

1 Fu ES, Tummala RP: Neuroprotection in brain and spinal cord trauma. Curr Opin Anaesthesiol 2005;18:181-187.

2 Tie G, Dai Y, Guan C, Zhu D, Song B: Research on compensation of suction deformation error of potassium dihydrogen phosphate crystal. Appl Opt 2013;52:110-116.

$>3$ Leal-Filho MB: Spinal cord injury: From inflammation to glial scar. Surg Neurol Int 2011;2:112.

- 4 David S, Kroner A: Repertoire of microglial and macrophage responses after spinal cord injury. Nat Rev Neurosci 2011;12:388-399.

5 Nesic O, Svrakic NM, Xu GY, McAdoo D, Westlund KN, Hulsebosch CE, Ye Z, Galante A, Soteropoulos P, Tolias P, Young W, Hart RP, Perez-Polo JR: DNA microarray analysis of the contused spinal cord: Effect of NMDA receptor inhibition. J Neurosci Res 2002;68:406-423.

6 Bareyre FM, Schwab ME: Inflammation, degeneration and regeneration in the injured spinal cord: Insights from DNA microarrays. Trends Neurosci 2003;26:555-563.

-7 Yunta M, Nieto-Diaz M, Esteban FJ, Caballero-Lopez M, Navarro-Ruiz R, Reigada D, Pita-Thomas DW, Del AA, Munoz-Galdeano T, Maza RM: MicroRNA dysregulation in the spinal cord following traumatic injury. Plos One 2012;7:e34534.

8 Alvarez-Garcia I, Miska EA: MicroRNA functions in animal development and human disease. Development 2005;132:4653-4662.

9 Lee RC, Feinbaum RL, Ambros V: The C. Elegans heterochronic gene lin-4 encodes small RNAs with antisense complementarity to lin-14. Cell 1993;75:843-854.

10 Cao X, Pfaff SL, Gage FH: A functional study of miR-124 in the developing neural tube. Genes Dev 2007;21:531-536.

11 Strickland ER, Hook MA, Balaraman S, Huie JR, Grau JW, Miranda RC: MicroRNA dysregulation following spinal cord contusion: Implications for neural plasticity and repair. Neuroscience 2011;186:146-160.

12 Liu G, Detloff MR, Miller KN, Santi L, Houle JD: Exercise modulates microRNAs that affect the PTEN/mTOR pathway in rats after spinal cord injury. Exp Neurol 2012;233:447-456.

13 Wang CX, Reece C, Wrathall JR, Shuaib A, Olschowka JA, Hao C: Expression of tumor necrosis factor alpha and its mRNA in the spinal cord following a weight-drop injury. Neuroreport 2002;13:1391-1393.

14 DeVivo MJ, Go BK, Jackson AB: Overview of the national spinal cord injury statistical center database. J Spinal Cord Med 2002;25:335-338. 


\section{Cellular Physiology Cell Physiol Biochem 2018;51:1921-1931

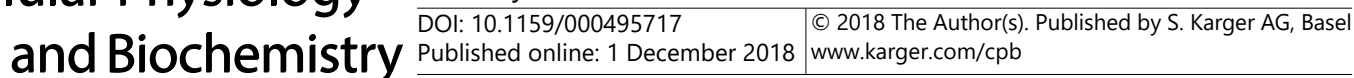 \\ Yang et al.: miR-544a is Involved in the Development of Spinal Cord Injury}

15 Anderson KD, Abdul M, Steward O: Quantitative assessment of deficits and recovery of forelimb motor function after cervical spinal cord injury in mice. Exp Neurol 2004;190:184-191.

-16 Otaegi G, Pollock A, Sun T: An optimized sponge for microRNA miR-9 affects spinal motor neuron development in vivo. Front Neurosci 2011;5:146.

$>17$ Cao X, Pfaff SL, Gage FH: A functional study of miR-124 in the developing neural tube. Genes Dev 2007;21:531-536.

18 Sehm T, Sachse C, Frenzel C, Echeverri K: MiR-196 is an essential early-stage regulator of tail regeneration, upstream of key spinal cord patterning events. Dev Biol 2009;334:468-480.

19 Tsitsiou E, Lindsay MA: MicroRNAs and the immune response. Curr Opin Pharmacol 2009;9:514-520.

20 Harris TA, Yamakuchi M, Ferlito M, Mendell JT, Lowenstein CJ: MicroRNA-126 regulates endothelial expression of vascular cell adhesion molecule 1. Proc Natl Acad Sci U S A 2008;105:1516-1521.

21 Tetreault LA, Kopjar B, Vaccaro A, Yoon ST, Arnold PM, Massicotte EM, Fehlings MG: A clinical prediction model to determine outcomes in patients with cervical spondylotic myelopathy undergoing surgical treatment: Data from the prospective, multi-center AOSpine North America study. J Bone Joint Surg Am 2013;95:1659-1666.

-22 Yanaka Y, Muramatsu T, Uetake H, Kozaki K, Inazawa J: MiR-544a induces epithelial-mesenchymal transition through the activation of WNT signaling pathway in gastric cancer. Carcinogenesis 2015;36:1363-1371.

23 Lu P, Gu Y, Li L, Wang F, Qiu X: MiR-544a promotes breast cancer cell migration and invasion reducing cadherin 1 expression. Oncol Res 2016; 23: 165-170.

24 Ma L. MicroRNA and metastasis. Adv Cancer Res 2016;132:165-207.

25 Zhi Q, Guo X, Guo L, Zhang R, Jiang J, Ji J, Zhang J, Zhang J, Chen X, Cai Q, Li J, Liu B, Zhu Z, Yu Y: Oncogenic miR-544 is an important molecular target in gastric cancer. Anticancer Agents Med Chem 2013;13:270275.

26 Mo XM, Li HH, Liu M, Li YT: Downregulation of GSK3beta by miR-544a to maintain self-renewal ability of lung caner stem cells. Oncol Lett 2014;8:1731-1734.

27 Pineau I, Sun L, Bastien D, Lacroix S: Astrocytes initiate inflammation in the injured mouse spinal cord by promoting the entry of neutrophils and inflammatory monocytes in an IL-1 receptor/MyD88-dependent fashion. Brain Behav Immun 2010;24:540-553.

-28 Perrin FE, Lacroix S, Aviles-Trigueros M, David S: Involvement of monocyte chemoattractant protein-1, macrophage inflammatory protein-1alpha and interleukin-1beta in Wallerian degeneration. Brain 2005;128:854-866.

29 Yokoyama M, Nishi Y, Miyamoto Y, Nakamura M, Akiyama K, Matsubara K, Okubo K: Molecular cloning of a human neuroD from a neuroblastoma cell line specifically expressed in the fetal brain and adult cerebellum. Brain Res Mol Brain Res 1996;42:135-139. 